\title{
Review
}

Neuropsychobiology

\section{Multimodal EEG Recordings, Psychometrics and Behavioural Analysis}

\author{
Peter H. Boeijinga \\ International Pharmaco-EEG Society, Berlin, Germany
}

\begin{abstract}
Key Words
Visual oddball, P300-informed · Functional magnetic resonance imaging $\cdot$ Independent component analysis, blood oxygenation level dependent . Electroencephalography · Magnetoencephalography . Neuropharmacology · Sensory activation · Higher mental function
\end{abstract}

\begin{abstract}
High spatial and temporal resolution measurements of neuronal activity are preferably combined. In an overview on how this approach can take shape, multimodal electroencephalography (EEG) is treated in 2 main parts: by experiments without a task and in the experimentally cued working brain. It concentrates first on the alpha rhythm properties and next on data-driven search for patterns such as the default mode network. The high-resolution volumic distributions of neuronal metabolic indices result in distributed cortical regions and possibly relate to numerous nuclei, observable in a non-invasive manner in the central nervous system of humans. The second part deals with paradigms in which nowadays assessment of target-related networks can align level-dependent blood oxygenation, electrical responses and behaviour, taking the temporal resolution advantages of event-related potentials. Evidence-based electrical propagation in serial tasks during performance is now to a large extent attributed to interconnected pathways, particularly chronometry-dependent ones, throughout a chain including a dorsal stream, next ventral cortical areas taking
\end{abstract}

the flow of information towards inferior temporal domains. The influence of aging is documented, and results of the first multimodal studies in neuropharmacology are consistent. Finally a scope on implementation of advanced clinical applications and personalized marker strategies in neuropsychiatry is indicated.

(c) 2016 S. Karger AG, Basel

\section{Introduction}

Engineering and informatics parts of functional imaging are impressive, and one should attempt to bring closer the physiologist's view and intuitive notions to the sophisticated 3-dimensional (3-D) colour reconstructions. This article is meant to initiate the physiologist and psychologist, who know electroencephalography (EEG) and related techniques already, to enlarge their understanding of localized networks; applications and certain concepts of brain (in)efficacy, (aging) deficits or marker potential accompanying medication have been highlighted. Whenever relevant in this inventory, some other contributions to this special issue from International Pharmaco-EEG Society (IPEG) EEG enthusiasts are highlighted (cross-linked only).

A repeatedly recurring criticism is the fact that scalp EEG is partly the result of artefacts like muscle contamination or even fluid dynamics/rheology. The summated activity of neurons forms part of the theory of the EEG, and hence a known localization makes the rela-

\section{KARGER 125\%}

(C) 2016 S. Karger AG, Basel

0302-282X/16/0724-0206\$39.50/0

E-Mail karger@karger.com

www.karger.com/nps
Peter H. Boeijing

5 Impasse du Noyer

FR-68800 Roderen (France)

E-Mail peterboeijinga@ymail.com 
Fig. 1. Example of sLORETA tomography of alpha power (around $10 \mathrm{~Hz}$ ) for the difference between eyes closed - eyes open in axial (a) and sagittal (b) planes (scale for maximal current density $231 \mathrm{mV}$ ) for 1 individual volunteer. c MEG narrow-band filtered alpha band activity for 28 reconstructed sites out of 148 magnetometers from a WH2500 System (BTi, San Diego), aligned with the similarly filtered signal on C3 showing in-phase (inset O2)/antiphase (inset $\mathrm{Pz}$ ) patterns over the occipital domains; epoch length for insets $400 \mathrm{~ms}$. d An illustration of 'meshing' equidistant nodes for 'voxelizing' the EEG/MEG patterns in grey matter atlas.

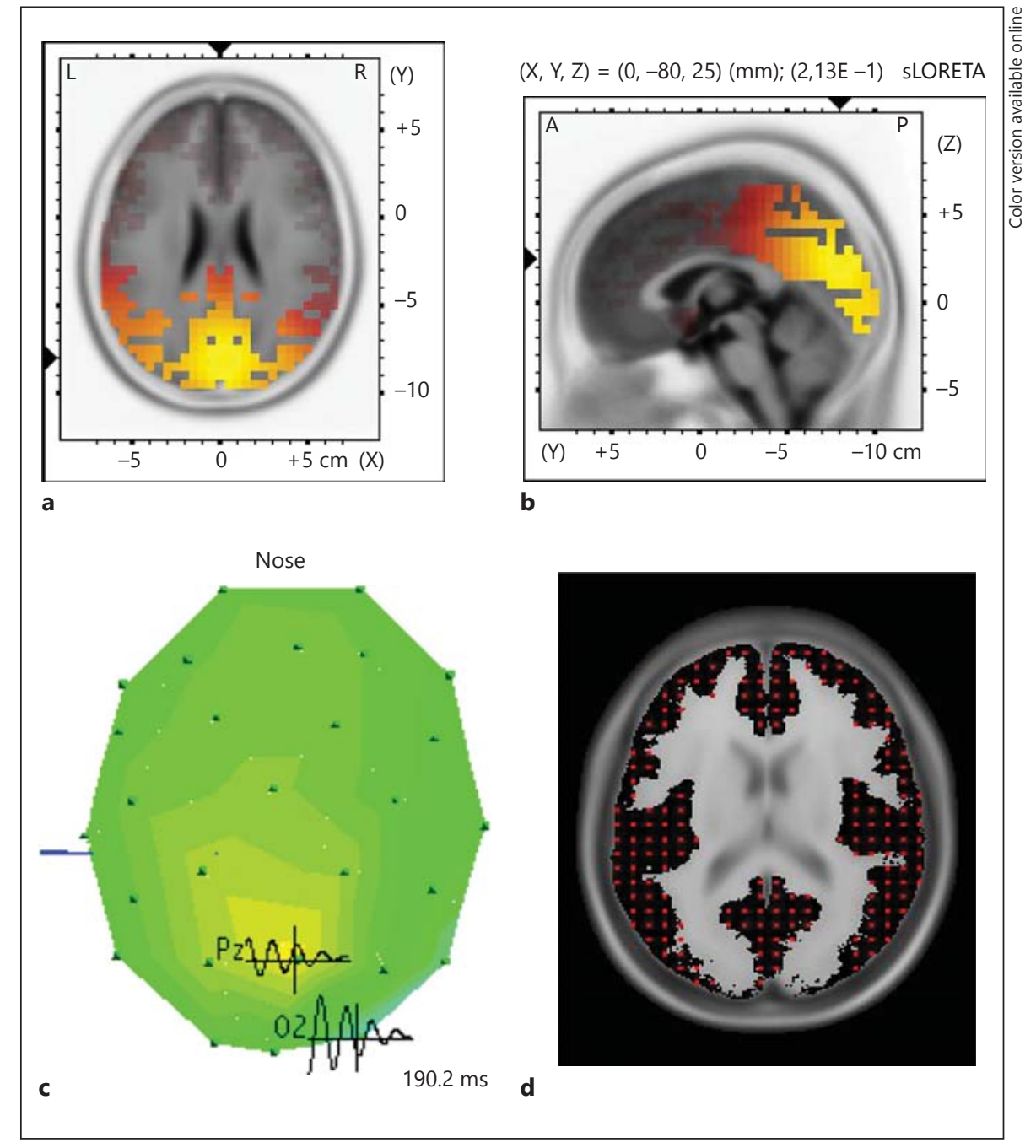

tionship between intracerebral mechanisms and scalp observations plausible [1]. There is great interest in high frequencies, but these are most susceptible to non-cerebral sources [2; Nottage and Horder, this issue]. Also an understanding of how drugs selectively act on neurotransmitter systems that perturb the EEG can be of help in the understanding of where targets in the brain are located. The long-standing tradition of EEG will not be highlighted here, and many details can be found in the IPEG guidelines $[3,4]$ and for instance in the paper on pharmaco-EEG [Jobert and Wilson, this issue].

\section{'Voxel-Wise' Imaging in EEG}

Plausible current source densities have been proposed by methods like sLORETA (standardized low-resolution brain electromagnetic tomography, available as freeware $[5,6])$, which attributes current densities from a popula- tion of neurons in a voxel-wise fashion to equidistant physical points of a 3-D raster that 'converts' scalp electrical patterns in current density distributions (with plausible assumptions on cytoarchitectonics for optimization); the algorithms display intensity from 6,239 cortical grey matter voxels. These have $5-\mathrm{mm}$ resolution within the brain volume, and the equidistant elements are aligned to the anatomical shape of the brain, most commonly a standardized average structural brain with labelled subdivisions, or a subject's own head. Examples of physiological conditions for eyes-open/eyes-closed differences are depicted in figure 1. This concerns a representative atlas space sLORETA image for 28 electrode alpha EEG reconstructions for 1 single female healthy volunteer of ongoing activity around $10 \mathrm{~Hz}$ and reveal sources in the Brodmann areas 17 and 18 with high current densities in the calcarine fissure and precuneus for the scalp-derived EEG alpha 
waves. In many publications, a view like looking through a transparent 'glass' brain (surface-rendered projections including sagittal interhemisphere plane) has been implemented for comprehensive topographic evaluations.

\section{Good Imaging Practice in Scanners}

Group comparisons are commonly undertaken to study drug effects; a recommendation is to perform this with subjects closing the eyes for at least 1 run during relaxed wakefulness $[3,7]$. A statistical aid is definitely (paired) $t$ statistic [8], hence for 2-sample testing the eccentricity between group means is converted into a $\mathrm{Z}$ score in each voxel. In the case of normal distributions, the threshold for $5 \%$ for differences would be a cut-off at 1.96. Some teams suggested a threshold of $t=2.4$ to prevent false-positive results due to multiple pairwise comparisons (namely each node in fig. 1d) and allow to accept changes corresponding to the $\mathrm{p}<0.01$ [9] level with $\mathrm{N}$ reaching infinity. For pragmatic sample sizes, elegant $t$ adjustment using surrogate data is displayed graphically (fig. 2) useful to align statistical power of null hypothesis rejection [10]. Moreover, for the physiologist's view it is taken for granted that all authors in cited publications have done their best to deal with artefact suppression, risk of alpha inflation, proper dipole estimates and finally classification rules to avoid false-positive results. Also, the aspect of pattern detection via multivariate factorial discriminants and state-of-the-art matrix algebra inevitably poses the risk for overlearning; stability of solutions requires more than 1 cohort before drawing conclusions on a novel cohort (for high-dimensional haemodynamic patterns, see Vincent et al. [11]; for clinical multiband quantitative EEG pattern methods like bootstrap or split-half validation, see Coburn et al. [12]). In case of doubt the reader is referred to details and discussion on shortcomings in the discussion provided by the corresponding authors. So generally speaking, appropriate data cleaning for head motion/respiratory movements, cardiac interferences and the number of volunteers/patients are no issue.

\section{Free Running Brain Metabolic and Electromagnetic Activity}

Magnetoencephalography (MEG) could be used to render more profound insights into apparent EEG patterns which are - literally - superficial. First of all spontaneous MEG rhythms reported represented the first whole-head characterization in the mid-nineties [13].

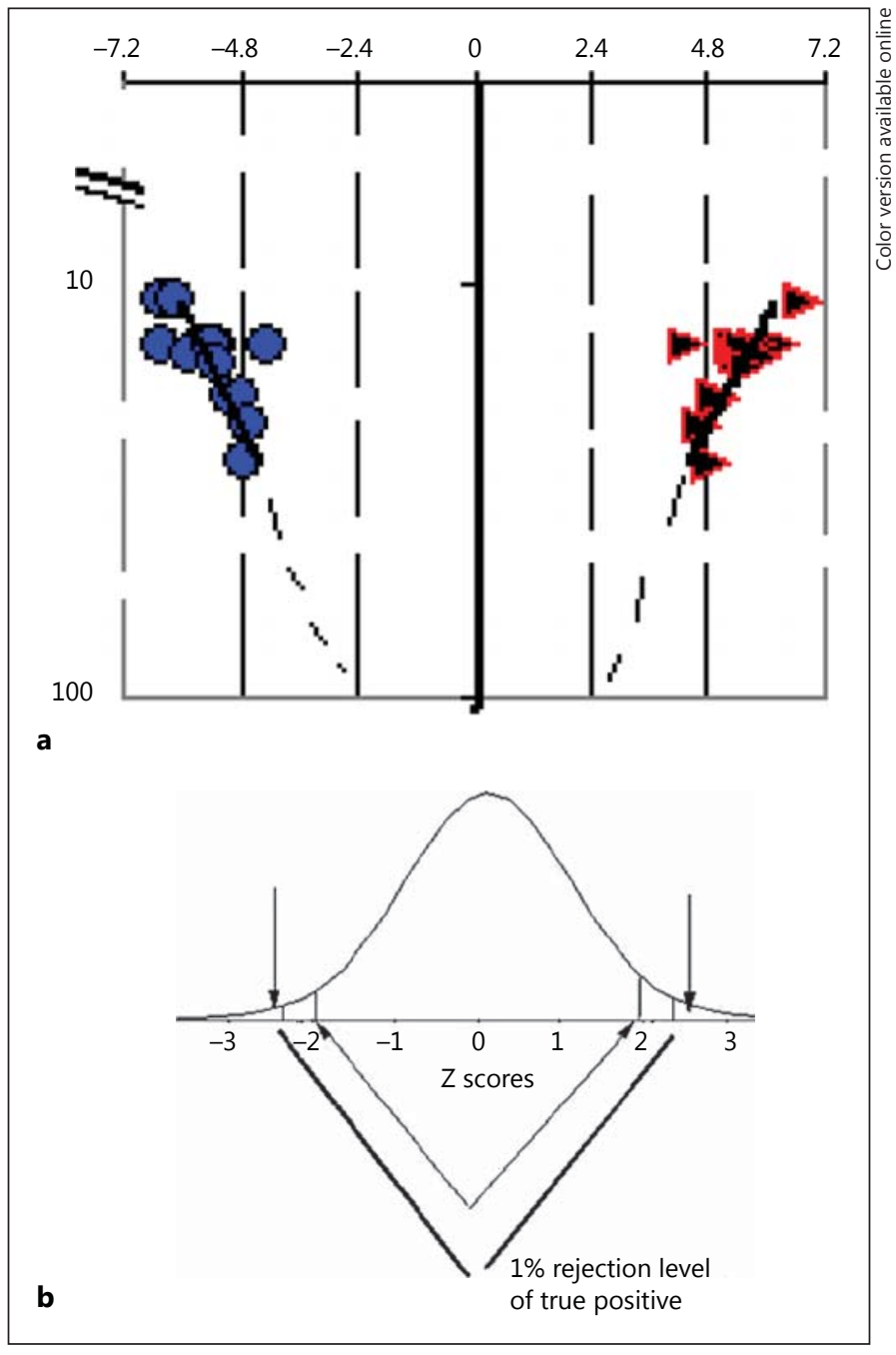

Fig. 2. a Iterative adjusted t values (horizontal axis) for paired comparisons as a function of sample size (vertical axis) on the basis of permutations [10] from in-house true pharmaco-EEG data from healthy volunteers. b Analytical distribution of normalized probabilities (surface is 1.00) for an infinite number of observations depicting pragmatic thresholds (horizontal axis as in a). Note that for changes in brain state (drug-induced or other) the $p<0.01$ level that corresponds to gaussian $\mathrm{t} \sim 2.3$ is attained for a sample size close to 100 .

The 7 volunteers (4 females) displayed mostly a strong presence of $10 \mathrm{~Hz}$ under eyes-closed conditions, and this desynchronizes when the subject opens the eyes.

The cortical localization corresponds to parieto-occipital lobe domains. Examples from my laboratory of the scalp distribution and average traces of ongoing alpha rhythm are shown in figure 1c, and the differential time shift of minima/maxima in the time course for 2 traces makes a 
generator mechanism plausible, like under resting conditions. Indeed dipole estimators of spontaneous oscillatory data segments were peaking at $20 \mathrm{~mm}$ under the skull (unaveraged helmet space data [13]), in line with an active layer of alpha generators, like in animal studies (beagles [14], awake macaques [15]). Of interest, when GABAergic transmission was globally enhanced by administration of benzodiazepine drugs at a dose of $\sim 2.5 \mathrm{mg}$, the clinical dose used to treat anxiety and sleep disorders given intravenously, localize MEG alpha frequency band changes (namely a full suppression) in exactly similar occipital regions suggesting that the generators are indeed under control of central nervous system (CNS)-inhibitory synapses [16]. Despite the use of 6,000 voxels per hemisphere (functional magnetic resonance imaging, $\mathrm{fMRI}$, constrained nodes in fig. 1d), the localized drug effects are said to be of low resolution.

So far there is good similarity with the EEG (e.g. theoretical framework [17-19]) but the point is that a gain became reality soon after using neuromagnetic recordings in the laboratory of Llinas and colleagues [20] who put in register reliably overlays of anatomy and processing of MEG data. Hence researchers could localize physiological data by performing topological adaptation of mapping results keeping fiducial points as landmarks. Indeed, the tonotopic organization of the auditory cortex in man studied with MEG was considered like this [21], and with Lu et al. [22] a beginning was made to associate the source locations with the details of brain morphology in humans. Before that, Freeman [23] projected putative sources of the $40-\mathrm{Hz}$ EEG rhythms on histological anatomical sections of the pyriform cortex in rabbits and constructed isocontours superimposed over the anatomical structure.

Whereas brain perfusion studies by the multidisciplinary team of Schulman et al. [24] noted a decrease during a cognitive challenge in frontal domains and also in the resting state, blood flow and oxygen consumption provide a pattern in different cortical regions that is not unpredictable, named default mode network [25]. In the meantime fMRI has been introduced to measure oxygen supply that serves to a great extent as fuel for neuronal activity $[26,27]$. Brain perfusion is nowadays also studied using a diffusible tracer, namely net magnetization of the blood water [28]. Low-frequency fMRI has been shown to give rise to a multitude of patterns in different parts of the brain, which seems reproducible.

It is intended here to show some great lines of how imaging techniques of relatively slow blood oxygenation level-dependent (BOLD) activity align to maps of EEG/ MEG phenomena occurring at the speed of neurons. The first striking example treated here in which multimodal- ity of brain investigations has been successful has been whole-brain fMRI or multi-voxel BOLD results directly related to occipital scalp montage for EEG recording. The application is the innovative cross-linking between distant or remote CNS divisions to put in evidence the thalamocortical interface in alpha frequency band generators. Out of a global scalp montage, $\mathrm{O} 1, \mathrm{Oz}$ and $\mathrm{O} 2$ have been used to measure spectral properties of the resting EEG. In parallel BOLD measures were coregistered using whole-head fMRI. An additional run during a visual task, namely checkerboard reversal, was carried out to localize the lateral geniculate nucleus of the thalamus.

For the resting state, the temporal fluctuations in occipital alpha rhythms from the EEG were reported to have positive correlations with thalamic regions. This is fully in line with partialized correlations, but measured in a highly invasive manner [29]. In the human study the lateral geniculate nucleus yielded no significant role in the regressor outcomes, and the electrical brain activity around $10 \mathrm{~Hz}$ seems to relate to metabolic activity in nuclei that were not activated by visual stimuli. The main result unveiled a pulvinar/calcarine network that distinguishes itself from functional connectivity for e.g. the mediodorsal nucleus that seems to form a resting state network (RSN) with anterior cingulate structures, indeed not supporting a relationship with alpha activity [30].

\section{What Is an RSN?}

This notion is based on observations focused on very low frequencies in BOLD imaging. Independent component analysis (ICA, 'principal component-like' discriminant), used as a blind separator and fully data-driven, has unveiled the existence of preferential patterns. BOLD patterns greatly overlap a default mode network $[25,31]$ as cited above for blood flow, and there have been reports on thalamic involvement as well [32]. The factorial mathematical ICA-BOLD technique is used to 'parcellate' patterns in the cortex domains [for an overview, see 33], which lends support to an idea that these form an underlying organizational principle at rest. Sketches of the presence of metabolic RSN patterns could have a link with oscillatory properties of electrically measured brain waves also mainly obtained in layered neo- and archicortex [34]. Such precise RSNs concern e.g. frontoparietal loops or ventral streams $[11,33]$ and can have overlap with positron emission tomography [35]. Of the multiple independent observations there are preliminary indications of the brain's functional organization combining 2 modalities: scalp EEGs were coregistered in the scanner and decomposed in global measures of delta, theta, alpha, beta and 
Fig. 3. Example of localization of gamma oscillatory EEG content for the eyes open/ eyes closed comparison in a group of 19 subjects (a) using sLORETA statistical comparisons by voxel-wise paired $t$ test performed on the mean (insula adjusted $\mathrm{t}=$ 4.1, $\mathrm{p}<0.05$ two-tailed) and MEG global alignment (b) of positive peak on C3 (cursor position) traces showing phase/antiphase patterns over the 2 hemispheres.

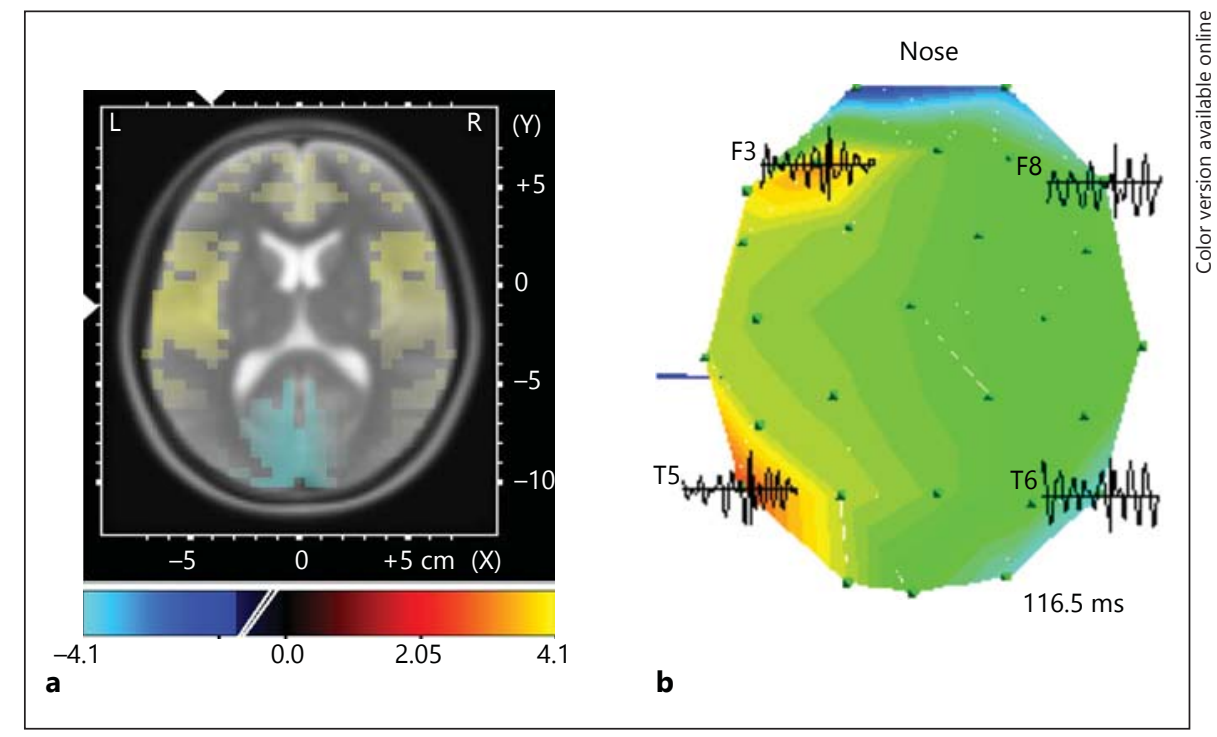

finally the (small magnitude) gamma waves. As expected the passive retinotopic RSN (No. 3 [32]) that includes the occipital domains had the highest positive correlations for the alpha frequency band.

\section{MEG and Oscillating Neuronal Currents}

Whole-head MEG data unveiled data-driven patterns of interdependence for remote cortical structures timeby-time in 5-min assessments. Overlap with the spatial variations between remote structures seen with fMRI (BOLD in a separate session) was present [36]. The team studied classical frequency bands and emphasized for the beta band reproducible volumic patterns representing the left and right lateral frontoparietal network probably involved in attention, medial parietal regions, sensorimotor network and visual network, and discerned in the neocortex the frontal lobes including the anterior cingulate cortex. Voxel-wise conjunction computations allowed to align temporal correlation maps for different volumic seeding references (relevant local source; for seed determination in connectivity, see Huster et al. [37]).

Similar results have been obtained by others for broadband high-resolution MEG and seem to stress that for both imaging modalities the solutions in the source space covary between $\mathrm{AMRI}$ and MEG suggesting that ion transport in neurons has - at least in part - a similar endogenous variation pattern as fMRI, supporting the idea that spatial BOLD patterns spread via axonal ramifications or, in other words, long-range connectivity related to neuronal fingerprints $[33,38]$.
Figure 3a displays group comparisons for the gamma frequency band having salient current densities or gamma sources in the left and right insula. This is a non-sensory domain and could point towards involvement in a network involved in e.g. attentional/mental function. An example of traces in the frequency band between 33 and $48 \mathrm{~Hz}$ is shown in figure $3 \mathrm{~b}$, again as sensor space topographic maps aligned to the MEG traces of the C3 site. The message is that indeed the topographic distribution is different from that for e.g. the alpha frequency band regardless of task. Paradigms designed to challenge repetitive task-related brain states is used for the notion of synchronization (called phase coherence) in near or remote cortical structures.

Within the resting network, correlations are sometimes carried out in multimodal imaging. EEG topographical distributions issued for the gamma band are shown in figure 4 and represent a first-order site-by-site connectivity. Amplitude of the real and imaginary complex parts remains constrained to the sensor space reference hemisphere (fig. 4a: C3 on the left, fig. 4b: C4 on the right). Indeed, pairwise correlations from the previously cited MEG-fMRI study showed MEG volumic significance for high frequencies with ipsilateral dominance, for example relative to a reference point, and only after overlays of the left and right seed computation they match fairly reliably the fMRI resting state networks (see fig. 2C in Brookes et al. [36]). The phase lag topography for gamma bands (fig. 4c: paired to C3, fig. $4 \mathrm{~d}$ : paired to C4) was widespread, and this is maybe more reminiscent of covariances in ICA-BOLD patterns. There is 


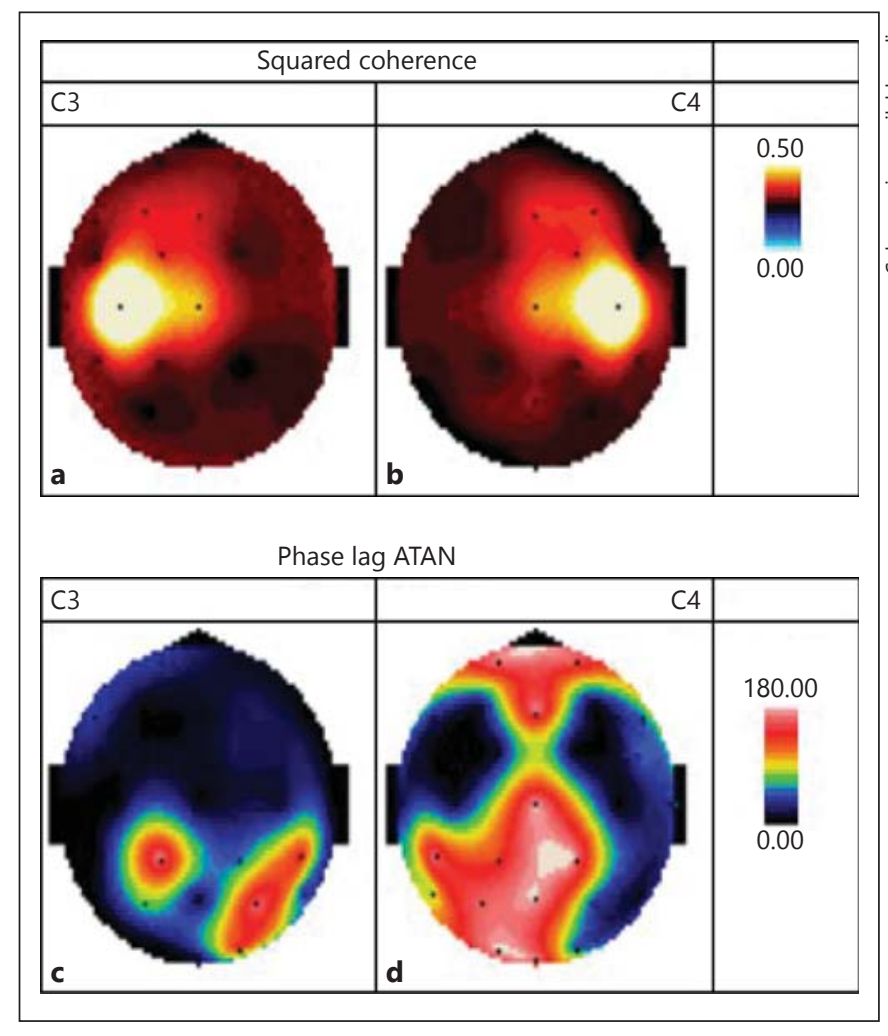

Fig. 4. Interpolated mapping of pairwise coherence calculations on source derivation (non-monopolar) EEG epochs in a 28-electrode montage 'seeded' with respect to electrode $\mathrm{C} 3$ (a) and with respect to electrode C4 (b). c, d Same as above but for the average phase difference. Note the bilateral spread of constant phase (dark zone equals difference at or near zero).

complementarity to resting fMRI connectivity of the widespread sensor space phase generalization in multielectrode EEG. Functional implications and pitfalls are presented later for neural synchronization in the working brain section below.

\section{The Working Brain}

At the functional level the RSNs are possibly a prerequisite for normal behaviour, and researchers associate internal processing/task control to RSN No. 1, dorsal attention to No. 2 and the senses, namely relaying and conveying visual information to No. 3 , auditory/phonological to No. 4 and somatosensory to No. $5[32,35]$ plus a ventral stream (No. 6) for endogenous mental activity [32].

It is impossible to cope in this review with all of these items, but some priority is given to the visual system and the putative chain of structures needed in self-referential mental function (No. 6).

Researchers in the field of electrical and neuromagnetic recordings noted for many years structured neuroelectric patterns, embedded in the EEG, visible as transient modifications time locked to cognitive, motor or sensory processing. The term electropsychopharmacology has been employed $[39,40]$ to indicate the applications as complement to behavioural studies trying to find functional correlates of mental function-related networks.

Particularly in the realm of clinical psychopharmacology, but also mental disorders, the use of interactive continuous performance tasks and simultaneous measurement of cognitive event-related potentials (ERPs) has established a suitable international consensus such as the allocation of attentional resources at appropriate timing or instantaneous dynamics of neural networks related to the task [41-45]. An example of a response shape displaying a maximum of cognitive response is shown in figure $5 \mathrm{a}$ with the aim to demonstrate that the continuous performance task family represents experimentally controllable steps [46] of information processing via electrical activity in e.g. a 2-choice visual oddball task. Indeed, reducing neuronal excitability by rufinamide [47] reduced the P3b between 290 and 420 ms ('anti-glutamate-like', see Frodl-Bauch et al. [48] and Umbricht et al. [49], for global drug effects Nieuwenhuis et al. [50] and Polich and Criado [51]).

When measured in the scanner, ICA components from 32 simultaneously recorded EEG electrodes best describing the deflection between 300 and 400 ms were shown to be associated with broad involvement of BOLD patterns in the RSN No.1, 2 and 3 (core network [52]) as well as occipital cortex and temporal-occipital regions dedicated to visual processing (RSN No. 3). Early steps of information processing in response to targets in this oddball paradigm align only with RSN No. 4 [33, see time scale in fig. 5]. The gradual change in invoking RSN in the course of chronometry also lends support to a neural signature in the endogenous topographies of ongoing BOLD signals [26].

Source space solutions for EEG processing mean in simple terms the creation of a virtual electrode that records intracranial electrical activity ('beamformer-like' [53]). The regional sources give as derived metric an output in nano-Ampere meter (nAm like MEG) and were hypothesized to match the time course of a dorsal attention cluster, and results comprise the inferior and posterior parietal lobe based on fMRI results from a reference cohort performing a 3-stimulus visual attention task. Confined EEG obtained during the same task 
Fig. 5. a Grand mean P300 response in an 'A-X-type' visual oddball task [49] with mental counting in a 2 -choice visual oddball task of targets $2 \mathrm{~h}$ after administration of rufinamide to dampen global excitability; the curve with the grey surface represents the drug-sensitive part of the signal. b Example of the time course of phasic gamma band response embedded in single event signals emerging after bandpass filtering; although not from the same study, the $\mathbf{x}$-axes have been aligned with panel $\mathbf{a}$. Filtered evoked reponses show nano-amplitude fast frequency oscillations in the gamma band $(\sim 40 \mathrm{~Hz})$. Horizontal axis: post-stimulus time in milliseconds; vertical axis: amplitude in microvolts (3 microvolts/tick in a).

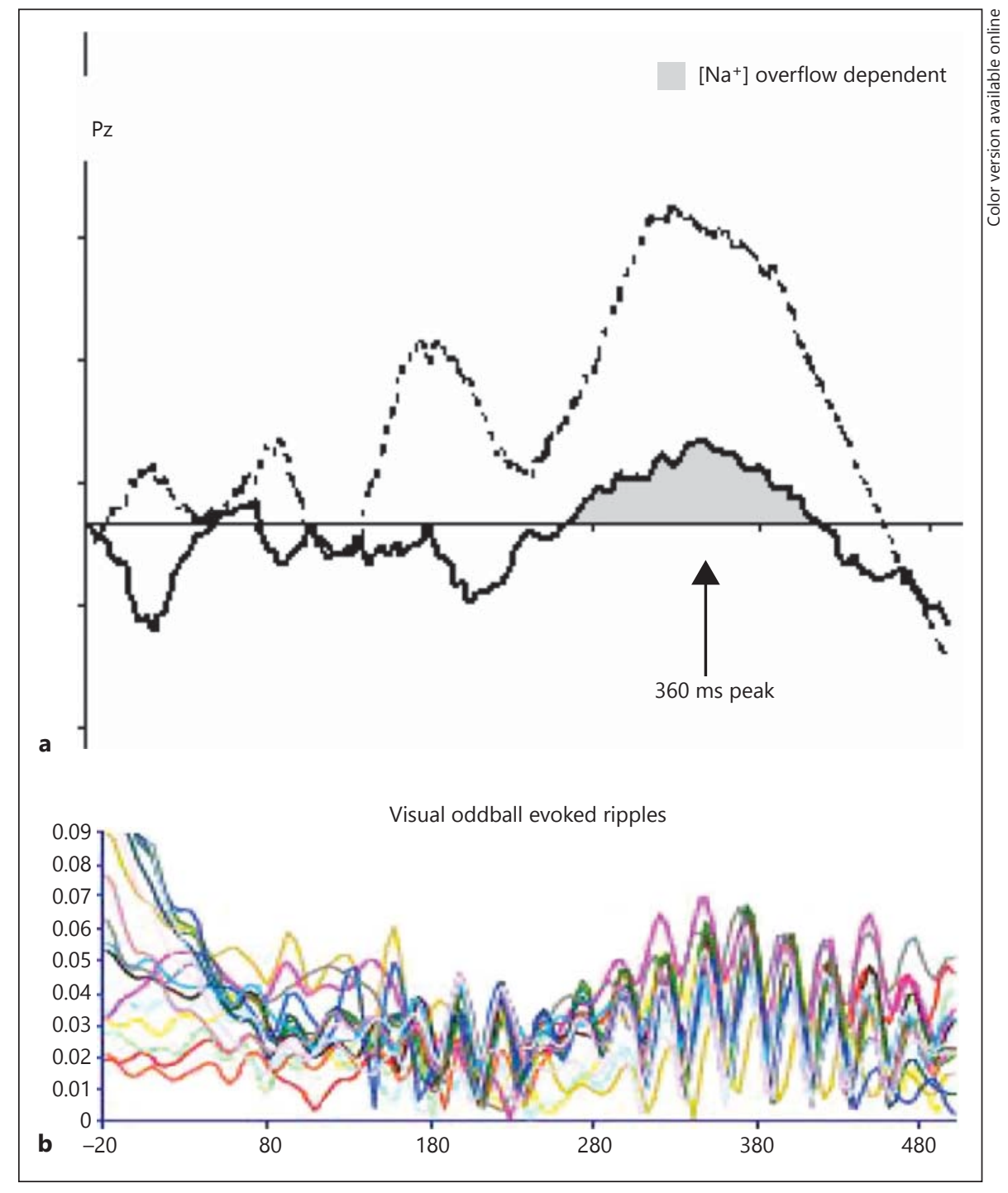

unveiled that these sources peaked between 400 and $600 \mathrm{~ms}$ for target and distractor sources as expected (60 channel derivations [54]). More ventrally the inferior temporal sources followed a similar time course with generally the distractor response peaking earlier than the attended target. Taken together the results are in line with temporoparietal topographic outcomes $[55,56]$ and stress the utility of the multimodal approach in the chronometry of a meaningful/plausible stimulus-response relationship of higher mental function [54] in a defined network.

\section{EEG P300 Amplitude-Informed fMRI}

To study the effect of nicotine in an oddball task with repeated brief checkerboard stimuli, the EEG P300 am- plitude-informed fMRI to deviants was used. In a group of males/females heterogeneous for smoking status, it was concluded that the direction of change in BOLD in a predetermined network that encompassed clusters like the anterior cingulate cortex, middle frontal gyrus, frontal orbital cortex, superior frontal gyrus and frontal pole during a nicotine session relative to (drug-free) baseline was correlated with the behavioural change; RT shortening is associated with a decrease in BOLD, whereas slowing induces increased BOLD [57]. A replication of nicotine administration in smokers had on average RT changes (by $-16 \mathrm{~ms}$ ) too small to decrease BOLD relative to baseline; when regressors based on P300 amplitude (that had a tendency to induce $\mathrm{P} 300$ response enhancement in a visual 'oddball' task) were invoked, BOLD di- 
minished significantly in the middle frontal gyrus and in addition the middle temporal gyrus [58]. Moreover, the RT effect was reminiscent of smoking 1 cigarette containing $1.1 \mathrm{mg}$ of nicotine [59, table 1 in 56].

Finally, with checkerboard oddballs, P300 in the intact target-related network included BOLD contributions in the anterior cingulate cortex, frontal cortex and temporoparietal cortical fields and atlas-based ERP tomography in addition to parieto-occipital current densities. Ketamine (active enantiomer $0.1 \mathrm{mg} / \mathrm{kg}$ plus 0.93 $\mathrm{mg} / \mathrm{kg} / \mathrm{h}$ ) reduced ERP by $71 \%$ in healthy volunteers; multimodal recordings showed that in the network depicted above on ketamine significantly fewer voxels emerged in both the BOLD as well as sLORETA 3-D volumic imaging outputs [60]. These authors did apply regressors of clinical performance scales that significantly worsened by $9 \%$ on single-trial BOLD ketamine effects. Whereas placebo data showed the involvement of parietal cortical areas (cuneus) in oddball performance, this was abolished by the drug. This experimental example of an 'accuracy-related fMRI' network lends support to the interpretation that this set of structures is involved in stimulus evaluation.

Without going into too much detail, oddball responses using sounds follow the same principles for drug effects [48] and disease [61, 62]. Moreover evidence is available from whole-head MEG assessments that the peak effects rely on the involvement of the hippocampus $[63,64$; for surface-rendered track profiles, see 41].

Mulert et al. [65] applied sLORETA techniques in male and female volunteers between 20 and 61 years of age in whom electrical patterns were recorded alone. Atlas space current density distributions yielded tomographic maxima in current strength in neuronal circuits being highest in the posterior cingulum and the precuneus, and comprise also other deep structures such as medial frontal and parietal cortex, inferior parietal lobule, the posterior and middle part of the superior temporal gyrus, and the dorsolateral and ventrolateral prefrontal cortex. For a separate study tomographic patterns were in line [66] but surprisingly evidenced a contribution in the supplementary motor area $[65,67]$.

Reports on fMRI-informed source modelling performing a 3-tone auditory oddball task are worth mentioning in this respect. The ERPs showed specific components to novelty and to predetermined targets; the multimodal results confirm the highly distributed engagement ranging from ventrolateral frontal and precentral cortical fields towards parietal cortical domains and caudate nucleus/putamen as well as thalamic nuclei $[68,69]$. For novelty the pattern did not fully overlap but the middle temporal gyrus was lighting up in this paradigm.

Age dependency for P3b was fully in line, and peak latency could be delayed as much as $60 \mathrm{~ms}$, relative to young participants [70]. Therefore using single-trial analysis applying regressors to put in register electrical optimal P300 sweeps and BOLD images in 2 age groups confirms the chronometry of higher mental function, and the anatomical support comprises indeed cortical structures like anterior cingulate and frontal domains as well as the temporoparietal junction covering the hippocampal region $[65,71]$.

Overall one can nowadays pinpoint activity in pathways relevant for higher mental function measured in multimodal (multiple choice [72], recollection of own thought [73]) or unimodal MEG assessments (sensory conflict [74], 'linguistic recognition' [75]) or combined with 'beamformer-like' analyses for tasks implying set shifting [76, 77].

The similarity of CNS networks treats visual oddballs during walking relatively similarly as when immobile in a laboratory environment [78] and the fact that EEG/ fMRI studies with auditory 2-tone or 3-tone oddballs evidence processing in pathways that converge to the same networks allow to enlarge the notion of natural neurocognition supporting real-life physiological mechanisms.

\section{Neural Synchronization in Healthy Volunteers}

Patterns of coherent activity in sensory processing have been described between remote structures $[79,80]$, now documented as a representation of perceptual 'binding' or synchrony at $\sim 40 \mathrm{~Hz}$ when correctly perceiving bistable images (Mooney faces or masked dogs); high-frequency gamma band responses (GBRs) were reported not to reveal topographically distinct areas, whereas robust phase-locking value (PLV [81]) adjustments have occurred uniquely during steps of information processing in the sensor space analyses $[82,83]$. The technique is close to coherence calculations: instantaneous phase values are computed for equivalent time points across trials. The result is an averaged expression (named PLV, vectorial moments in the polar co-ordinate system) in such a way that phase constancy equals 1 , and random phase shifts equal 0 . When placing in an experimental psychology perspective, namely the theory on distributed information consolidation and e.g. spatial feature integration [84], the GBR results near 1 suggest that timing of neural events forms the underlying framework. An example of a higher mental task-induced oscillatory burst is shown in the lower panel of figure $5 b$, the quantitative characterization by time-frequency plots for a group of 8 subjects is shown in figure 6 a. 
Fig. 6. Examples of time-frequency approach and drug effects in a visual oddball task for control condition (a) that unveiled $40-\mathrm{Hz}$ patterns reaching significance in 8 volunteers between 250 and $400 \mathrm{~ms}$ and for lorazepam (b) at the dose of $1 \mathrm{mg}$ (note that the GBR of the cognitive response has been abolished).

Fig. 7. Example illustrating modern network theory of drug effects during the visual P300. White lines correspond to the significant $(\mathrm{p}<0.01)$ coupling compared to scrambled data for a 7-cycle wavelet of dominant frequency of $39 \mathrm{~Hz}$ centred at $360 \mathrm{~ms}$; only cases $10 \%$ of highest PLVs having $\pm 10^{\circ}$ from zero phase have been retranscribed. The topographic map corresponds to 'PLV synchrotopy': the control state (a) yields a widespread pattern without much structure, whereas $2 \mathrm{~h}$ after lorazepam (b; lora $1 \mathrm{mg}$; see fig. $5 \mathrm{~b}$ and $6 \mathrm{~b}$ ) a more deterministic connectivity (at least interelectrode) highlights preferably bilateral temporoparietal domains.
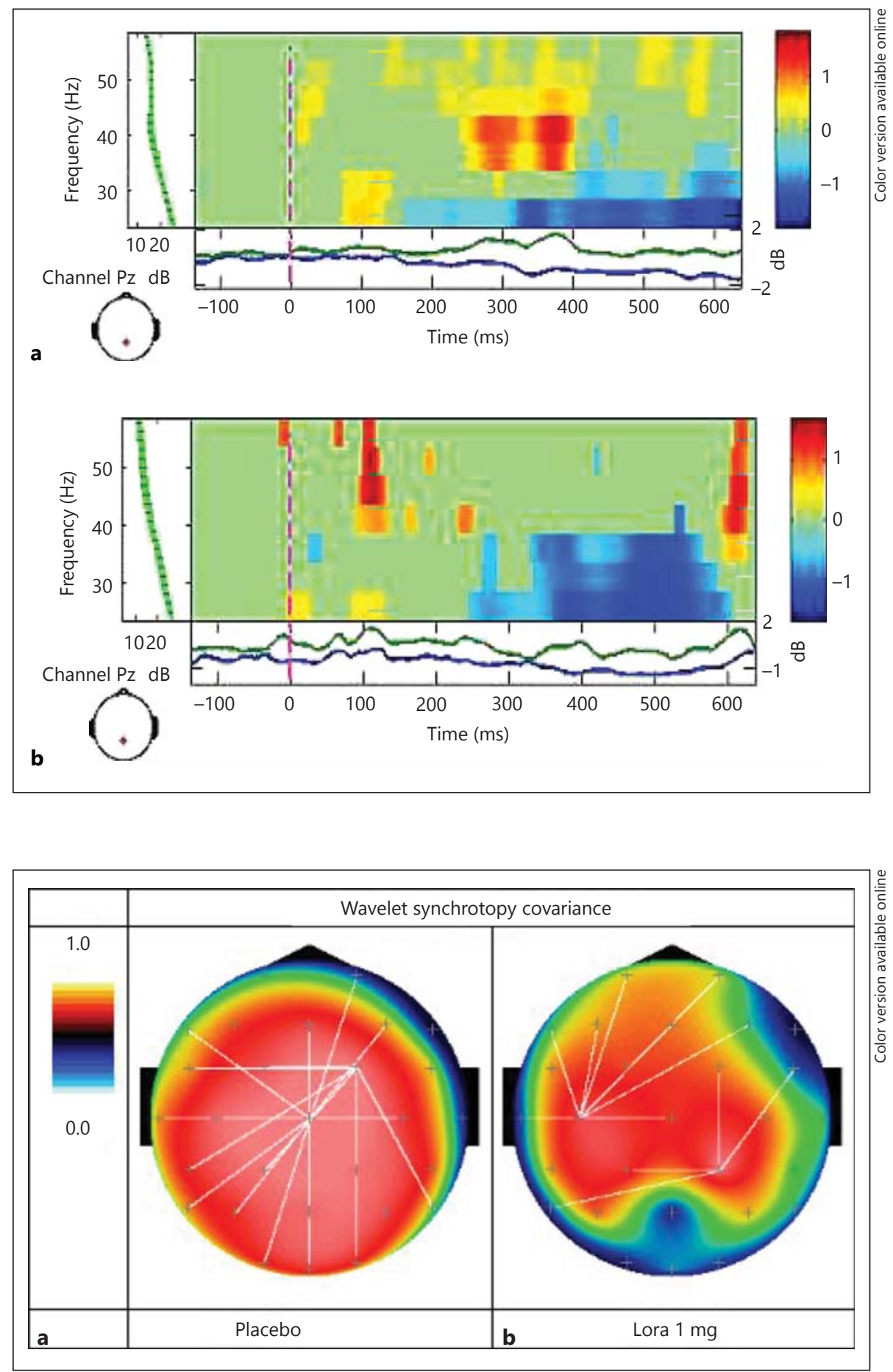

In my laboratory 2 doses of an attention-degrading drug were tested (lorazepam $1 \mathrm{mg}$ and $2 \mathrm{mg}$ ). Whereas the visual ERP shape was not significantly affected by the drug, the time-frequency content showed significant reduction in GBR bursts (fig. 6b). In other words this was associated with a tremendous re-organization in neural timing (fig. 7) maintaining an envelope amplitude in line with the evaluation of complex images mentioned above [82].

Unfortunately from these sensor space results one cannot deduce where in the brain the GBR has changed, but 
given the effects of a stimulant such as nicotine [58] an effect in the middle temporal gyrus would make sense. An example of a reference-free whole-brain pattern for gamma band neural synchronization can be seen in figure 7 and suggest diminished contributions in temporal regions. However, there is to date no consensus of the sources of these phasic high-frequency bursts [85, 86]. State-ofthe-art algorithms for late (cognition-related) GBR during a picture-naming task yielded a distributed pattern mainly in frontoparietal domains [87]. Despite high-density EEG, the authors just cited give an excellent review on caveats of signal-processing techniques and variability between different methods, keeping an uncertainty on the exact generators of GBR.

Calculations to characterize the whole-brain patterns to determine an average distance of significant connections (graph theory that yields path length [88]) known from modern network theory is applied in multimodal imaging but is beyond the scope of this paper.

Thanks to short-lasting wavelets, characterization by covarying oscillations having more or less constant phase angle differences (throughout repeated stimulus processing) is said to form a task-related functional process tracked in 1 region. Beyond that, one can also configure a matrix with filtered signals from distant regions during repeated processing on remote sites and see by an interrelationship if they both follow the stimulus in a coherent manner (neural connectivity concept or connectome). Principles of propagation and phase delay [89] as well a self-organized zero phase [90] are candidate modules for integrative CNS physiology to understand processing in the brain, and complexity becomes even more striking when taking into consideration the fact that task-related desynchronization can occur $[88,91]$.

\section{Conclusion and Outlooks}

To recapitulate, multimodal techniques reconcile experimentally controlled processing of large populations of neurons. Starting by resting conditions without a particular task, an initial flavour of covarying patterns emerges from the existing literature.

Probably the most promising methodology nowadays for understanding as many neurons as possible underlying brain function throughout performance and differences in processing is to employ 2 modalities; it has been demonstrated when taking the best of high spatial resolution (by haemodynamics or BOLD) and the best of high temporal resolution in single-trial event-related assess- ments from electrical potentials recorded from the scalp, decision-making networks in both visual and auditory discrimination have been mapped non-invasively.

Consistency exists in direction of effects of drugs and also aging.

There are shortcomings and mismatches as pointed out by Brookes et al. [36] for groups with patterns in one modality not having similarity in the other. Also inconsistencies could occur; for example, with benzodiazepines there is consensus that alpha band power decreases, but when longitudinal (sensor space) coherent activity was analysed in this band, no drug effect was found [92]. For these resting state and possible other observations there definitely is room for improvement.

Clever configuring machines and psychometric tests to fully understand (phase) synchronization issues in topdown, bottom-up and self-organized connectivity of remote brain structures make comprehensive brain physiology attainable. As such, ICA-BOLD patterns have, in part, guided where to look as is the case for MEG. The few examples of drug effects allow spending a few words on the biological psychiatry perspective.

Pathophysiology has for many decades been studied with brain scanning techniques, and attempts to combine methodologies revolutionize insight into the communities of scientists and medicine. One clinical example is the spectral centre of mass, used to characterize the severity of dysfunction in a population from society suffering from Alzheimer's disease. It was found that patient profiling gained in accuracy when using not only the spectra [93], but also connectivity $\left(\mathrm{cO}^{2}\right)$ information from MEG. Linear prediction of Alzheimer's Disease Assessment Scale - Cognition estimates was improved when adding a restricted number of well-chosen pairs across the 205-channel sensor array, to the centre-of-mass contribution alone [94]. Another example is the classification on the basis of a snapshot during resting MEG assessments (for dysrhythmia's classification studies, see [9597]). ERPs are proposed as disease markers with face validity in e.g. schizophrenia [98] or dementias [61, 62], and multimodal imaging could in the future definitely contribute to a consensus on construct validity. Given the overwhelming amount of interrelated or parallel/serial orchestrated neural patterns, one could contemplate the assessment of more and more unique deficits to each patient, in an attempt to determine populations and stratification in future drug trials avoiding excessive inhomogeneity in enrolled cohorts. 


\section{Acknowledgements}

The author wishes to express his gratitude to Drs. K. Mulligan and P. LeVan, who provided eye openers to the heavy problem of dual artefact rejection for getting multimodal imaging to work, Dr. R. Pascual-Marqui for sharing sLORETA and K. Redey-Puls for helping in editing the paper.

\section{Disclosure Statements}

The author has been an employee of a contract research organization performing commercial pharmaco-EEG and ERP studies, and held relevant positions in fundamental science departments.

\section{References}

1 Onton J, Makeig S: Information-based modeling of event-related brain dynamics. Prog Brain Res 2006;159:99-120.

$\checkmark 2$ Yuval-Greenberg S, Tomer O, Keren AS, Nelken I, Deouell LY: Transient induced gamma-band response in EEG as a manifestation of miniature saccades. Neuron 2008;58: 429-441.

>3 Jobert M, Wilson FJ, Ruigt GSF, Brunovsky M, Prichep LS, Drinkenburg WHIM, Babiloni $\mathrm{C}$, Boeijinga $\mathrm{PH}$, et al: Guidelines for the recording and evaluation of pharmaco-EEG data in man - International Pharmaco-EEG Society. Neuropsychobiology 2012;66:201220.

4 Michel CM, Murray MM: Towards the utilization of EEG as a brain imaging tool. Neuroimage 2012;61:371-385.

5 Pascual-Marqui RD, Michel CM, Lehmann D: Low resolution brain electromagnetic tomography: a new method for localizing electrical activity in the brain. Int J Psychophysiol 1994; 18; 49-65.

6 Pascual-Marqui RD: Standardized low-resolution brain electromagnetic tomography (sLORETA): technical details. Methods Find Exp Clin Pharmacol 2002;24(suppl D): 5-12.

7 Boeijinga PH, Calvi-Gries F, Demazieres A, Luthringer R: Planning of pharmacodynamic trials: specificities and possible solutions. II. Stability considerations under placebo and interpretation of drug effects on EEG. Methods Find Exp Clin Pharmacol 2002; 24(suppl C):17-26.

8 Salinsky MC, Oken BS, Storzbach D, Dodrill CB: Assessment of CNS effects of antiepileptic drugs by using quantitative EEG measures. Epilepsia 2003;44:1042-1050.

9 Marco-Pallarés J, Grau C, Ruffini G: Combined ICA-LORETA analysis of mismatch negativity. Neuroimage 2005;25:471-477.

10 Nichols TE, Holmes AP: Nonparametric permutation tests for functional neuroimaging: a primer with examples. Hum Brain Mapp 2002;15:1-25.

11 Vincent JL, Snyder AZ, Fox MD, Shannon BJ, Andrews JR, Raichle ME, Buckner RL: Coherent spontaneous activity identifies a hippocampal-parietal memory network. J Neurophysiol 2006;96:3517-3531.

12 Coburn KL, Lauterbach EC, Boutros NN, Black KJ, Arciniegas DB, Coffey CE: The val- ue of quantitative electroencephalography in clinical psychiatry: a report by the Committee on Research of the American Neuropsychiatric Association. J Neuropsychiatry Clin Neurosci 2006; 18:460-500.

13 Salmelin R, Hari R: Characterization of spontaneous MEG rhythms in healthy adults. Electroencephalogr Clin Neurophysiol 1994;91: 237-248.

14 Lopes da Silva FH, Storm van Leeuwen W: The cortical source of the alpha rhythm. Neurosci Lett 1977;6:237-241.

15 Bollimunta A, Chen Y, Schroeder CE, Ding M: Neuronal mechanisms of cortical alpha oscillations in awake-behaving macaques. J Neurosci 2008;28:9976-9988.

16 Ahveninen J, Lin F-H, Kivisaari R, Autti T, Hämaäläinen $M$, Stufflebeam $S$, et al: MRIconstrained spectral imaging of benzodiazepine modulation of spontaneous neuromagnetic activity in human cortex. Neuroimage 2007;35:577-582.

17 Ahlfors SP, Han J, Lin F-H, Witzel T, Belliveau JW, Hämäläinen MS, Halgren E: Cancellation of EEG and MEG signals generated by extended and distributed sources. Hum Brain Mapp 2010;31:140-149.

18 David O, Friston KJ: A neural mass model for MEG/EEG: coupling and neuronal dynamics. Neuroimage 2003;20:1743-1755.

19 Huotilainen M, Winkler I, Alho K, Escera C Virtanen J, Ilmoniemi RJ, Jääskeläinen IP, Pekkonen E, Näätänen R: Combined mapping of human auditory EEG and MEG responses. Electroencephalogr Clin Neurophysiol 1998; 108:370-379.

20 Yamamoto T, Williamson SJ, Kaufman L, Nicholson C, Llinas R: Magnetic localization of neuronal activity in the human brain. Proc Natl Acad Sci U S A 1988;85:8732-8736.

21 Romani GL, Williamson SJ, Kaufman L: Biomagnetic instrumentation. Rev Sci Instrum 1982;53:1815-1845.

22 Lu ZL, Williamson SJ, Kaufman L: Behavioral lifetime of human auditory sensory memory predicted by physiological measures. Science 1992;258:1668-1670.

23 Freeman WJ: Spatial properties of an EEG event in the olfactory bulb and cortex. Electroencephalogr Clin Neurophysiol 1978;44: 586-605.

24 Schulman GL, Fiez JA, Corbetta M, Buckner RL, Miezin FM, Raichle ME, Petersen SE:
Common blood flow changes across visual tasks. II. Decreases in cerebral cortex. J Cogn Neurosci 1997;9:648-663.

-25 Raichle ME, MacLeod AM, Snyder AZ, Powers WJ, Gusnard DA, Shulman GL: A default mode of brain function. Proc Natl Acad Sci U S A 2001;98:676-682.

-26 Blinowska KL, Müller-Putz G, Kaiser V, Astolfi L, Vanderperren K, Van Huffel S, Lemieux L: Multimodal imaging of human brain activity: rational, biophysical aspects and modes of integration. Comput Intell Neurosci 2009;813607:1-10.

27 Ogawa S, Menon RS, Kim S-G, Ugurbil $\mathrm{K}$ : On the characteristics of functional magnetic resonance imaging of the brain. Annu Rev Biophys Biomol Struct 1998;27:447474.

28 Setcharunpaisan P, Ramalho J, Castillo M: Arterial spin labeling in neuroimaging. World J Radiol 2010;2:384-398.

29 Lopes da Silva FH, van Lierop TH, Schrijer $\mathrm{CF}$, van Leeuwen WS: Organization of thalamic and cortical alpha rhythms: spectra and coherences. Electroencephalogr Clin Neurophysiol 1973;35:627-639.

- 30 Liu Z, de Zwart JA, Yao B, van Gelderen P, Kuo LW, Duyn JH: Finding thalamic BOLD correlates to posterior alpha EEG. Neuroimage 2012;63:1060-1069.

31 Greicius MD, Srivastava G, Reiss AL, Menon V: Default-mode network activity distinguishes Alzheimer's disease from healthy aging: evidence from functional MRI. Proc Natl Acad Sci U S A 2004;101:4637-4642.

32 Mantini D, Perrucci MG, Del Gratta C, Romani GL, Corbetta M: Electrophysiological signatures of resting state networks in the human brain. Proc Natl Acad Sci U S A 2007; 104:13170-13175.

33 Mantini D, Marzetti L, Corbetta M, Romani GL, Del Gratta C: Multimodal integrationn of fMRI and EEG data for high spatial and temporal resolution analysis of brain networks. Brain Topogr 2010;23:150-158.

34 Steriade M: Impact of network activities on neuronal properties in corticothalamic systems. J Neurophysiol 2001;86:1-39.

35 De Luca M, Beckmann CF, De Stefano N, Matthews PM, Smith SM: fMRI resting state networks define distinct modes of long-distance interactions in the human brain. Neuroimage 2006;29:1359-1367. 
-36 Brookes MJ, Woolrich M, Luckhoo H, Price D, Hale JR, Stephenson MC, Barnes GR, Smith SM, Morris PG: Investigating the electrophysiological basis of resting state networks using magnetoencephalography. Proc Natl Acad Sci U S A 2012;108:1678316788.

- 37 Huster RJ, Debener S, Eichele T, Herrmann CS: Methods for simultaneous EEG-fMRI: an introductory review. J Neurosci 2012;32: 6053-6060.

- 38 De Pasquale F, Della Penna S, Snyder AZ, Lewis C, Mantini D, Marzetti L, Belardinelli P, Ciancetta L, Pizzella V, Romani GL, Corbetta M: Temporal dynamics of spontaneous MEG activity in brain networks. Proc Natl Acad Sci U S A 2009;107:60406045.

39 Kenemans JL, Kähkönen S: How human electrophysiology informs psychopharmacology: from bottom-up driven processing to topdown control. Neuropsychopharmacol Rev 2011;36:26-51.

-40 Vilberg KL, Rugg MD: Functional significance of retrieval-related activity in lateral parietal cortex: evidence from fMRI and ERPs. Hum Brain Mapp 2009;30:14901501.

-41 Halgren E, Marinkovic K, Chauvel P: Generators of the late potentials in auditory and visual oddball tasks. Electroencephalogr Clin Neurophysiol 1998;106:156-164.

-42 Hillyard SA: Electrical and magnetic brain recordings: contributions to cognitive neuroscience. Curr Opin Neurobiol 1993;3:217224.

-43 Gehring WJ, Gratton G, Coles MG, Donchin E: Probability effects on stimulus evaluation and response processes. J Exp Psychol Hum Percept Perform 1992;18:198-216.

44 Michel CM, Thut G, Morand S, Khateb A, Pegna AJ, Grave de Peralta R, Gonzalez S, Seeck M, Landis T: Electric source imaging of human brain functions. Brain Res Brain Res Rev 2011;36:108-118.

45 Nieuwenhuis S, de Rover M: Event related potentials; in Stolerman I (ed): Encyclopedia PsychoPharmacol. Heidelberg, Springer, 2010, pp 503-507.

-46 Duncan CC, Barry RJ, Connolly JF, Fischer C, Michie PT, Näätänen R, Polich J, Reinvang I, Van Petten C: Event-related potentials in clinical research: guidelines for eliciting, recording, and quantifying mismatch negativity, P300, and N400. Clin Neurophysiol 2009; 120:1883-1908.

47 Arroyo S: Rufinamide. Neurotherapeutics 2007;4:155-162.

48 Frodl-Bauch T, Bottlender R, Hegerl U: Neurochemical substrates and neuroanatomical generators of the event-related P300. Neuropsychobiology 1999;40:86-94.

-49 Umbricht D, Schmid L, Koller R, Vollenweider FX, Hell D, Javitt DC: Ketamine-induced deficits in auditory and visual context-dependent processing in healthy volunteers: implications for models of cognitive deficits in schizophrenia. Arch Gen Psychiatry 2000;57: 1139-1147.

50 Nieuwenhuis S, Aston-Jones G, Cohen JD: Decision making, the P3, and the locus coeruleus-norepinephrine system. Psychol Bull 2005; 131:510-532.

51 Polich J, Criado R: Neuropsychology and neuropharmacology of P3a and P3b. Int J Psychophysiol 2006;60:172-185.

52 Mantini D, Corbetta M, Mauro G, Perrucci MG, Romani GL, Del Gratta C: Large-scale brain networks account for sustained and transient activity during target detection. Neuroimage 2009;44:265-274.

53 Luckoo HT, Brookes MJ, Woolrich MW: Multi-session statistics on beamformed MEG data. Neuroimage 2014;95:330-335.

54 Bledowski C, Prvulovic D, Hoechstetter K, Scherg M, Wibral M, Goebel R, Linden DE: Localizing P300 generators in visual target and distractor processing: a combined eventrelated potential and functional magnetic resonance imaging study. J Neurosci 2004;24: 9353-9360.

55 Comerchero MD, Polich J: P3a and P3b from typical auditory and visual stimuli. Clin Neurophysiol 1999;110:24-30.

56 Polich J, Kok A: Cognitive and biological determinants of P300: an integrative review. Biol Psychol 1995;41:103-146.

-57 Warbrick T, Mobascher A, Brinkmeyer J, Musso F, Stoecker T, Shah NJ, Vossel S, Winterer G: Direction and magnitude of nicotine effects on the fMRI BOLD response are related to nicotine effects on behavioral performance. Psychopharmacology 2011;215:333-344.

58 Warbrick T, Mobascher A, Brinkmeyer J, Musso F, Stoecker T, Shah NJ, Fink GR, Winterer G: Nicotine effects on brain function during a visual oddball task: a comparison between conventional and EEG-informed fMR analysis. J Cogn Neurosci 2012;24:16821694.

59 Houlihan ME, Pritchard WS, Robinson JH: Faster P300 latency after smoking in visual but not auditory oddball tasks. Psychopharmacology 1996;123:231-238.

60 Musso F, Brinkmeyer J, Ecker D, London MK, Thieme G, Warbrick T, Wittsack H-J, Saleh A, Greb W, de Boer P, Winterer G: Ketamine effects on brain function - simultaneous fMRI/EEG during a visual oddball task. Neuroimage 2011;58:508-525.

61 Olichney JM, Yang J-C, Taylor J, Kutas M: Cognitive event-related potentials: biomarkers of synaptic dysfunction across the stages of Alzheimer's disease. J Alzheimer Dis 2011; 26:215-228

62 Polich J, Corey-Bloom J: Alzheimer's disease and P300: review and evaluation of task and modality. Curr Alzheimer Res 2005;2:515525.

63 Nishitani N, Nagamine T, Fujiwara N, Yazawa S, Shibasaki H: Cortical-hippocampal auditory processing identified by magnetoencephalography. J Cogn Neurosci 1998; 10: 231-247.
64 Boeijinga PH: Objective markers of drug effects on brain function by way of scalp potential recordings in healthy volunteers. Dialogues Clin Neurosci 2002;4:388-394.

65 Mulert C, Pogarell O, Juckel G, Rujescu D, Giegling I, Rupp D, Mavrogiorgou P, Bussfeld P, Gallinat J, Möller HJ, Hegerl U: The neural basis of the P300 potential. Focus on the timecourse of the underlying cortical generators. Eur Arch Psychiatry Clin Neurosci 2004;254: 190-198.

-66 Anderer P, Saletu B, Semlitsch HV, PascualMarqui RD: Non-invasive localization of P300 sources in normal aging and age-associated memory impairment. Neurobiol Aging 2003;24:463-479.

-67 Mulert C, Jäger L, Schmitt R, Bussfeld P, Pogarell O, Möller HJ, Juckel G, Hegerl U: Integration of fMRI and simultaneous EEG: towards a comprehensive understanding of localization and time-course of brain activity in target detection. Neuroimage 2004;22:8394.

68 Menon V, Ford JM, Lim KO, Glover GH, Pfefferbaum A: Combined event-related fMRI and EEG evidence for temporal-parietal cortex activation during target detection. Neuroreport 1997;8:3029-3037.

-69 Strobel A, Debener S, Sorger B, Peters JC, Kranczioch C, Hoechstetter K, Engel AK, Brocke B, Goebel R: Novelty and target processing during an auditory novelty oddball: a simultaneous event-related potential and functional magnetic resonance imaging study. Neuroimage 2008;40:869-883.

-70 O'Connell RG, Balsters JH, Kilcullen SM, Campbell W, Bokde AW, Lai R, Upton N, Robertson IH: A simultaneous ERP/fMRI investigation of the P300 aging effect. Neurobiol Aging 2012;33:2448-2461.

71 Jückel G, Karch S, Kawohl W, Kirsch V, Jäger L, Leicht G, Lutz J, Stammel A, Pogarell O, Ertl M, Reiser M, Hegerl U, Möller HJ, Mulert C: Age effects on the P300 potential and the corresponding fMRI BOLD-signal. Neuroimage 2012;60:2027-2034.

72 Bledowski C, Cohen Kadosh K, Wibral M, Rahm B, Bittner RA, Hoechstetter K, Scherg M, Maurer K, Goebel R, Linden DE: Mental chronometry of working memory retrieval: a combined functional magnetic resonance imaging and event-related potentials approach. J Neurosci 2006;26:821-829.

73 Rugg MD, Vilberg KL: Brain networks underlying episodic memory retrieval. Curr Opin Neurobiol 2013;23:255-260.

74 Ukai S, Shinosaki K, Ishii R, Ogawa A, Mizuno-Matsumoto $\mathrm{Y}$, Inouye $\mathrm{T}$, Hirabuki N, Yoshimine T, Robinson SE, Takeda M: Parallel distributed processing neuroimaging in the Stroop task using spatially filtered magnetoencephalography analysis. Neurosci Lett 2002;334:9-12.

75 Härle M, Dobe C, Cohen R, Rockstroh B: Brain activity during syntactic and semantic processing - a magnetoencephalographic study. Brain Topogr 2002;15:3-11.
Multimodal EEG Recordings,

Psychometrics and Behavioural Analysis
Neuropsychobiology 2015;72:206-218 DOI: $10.1159 / 000437434$ 
-76 Bayless SJ, Gaetz WC, Cheyne DO, Taylor MJ: Spatiotemporal analysis of feedback processing during a card sorting task using spatially filtered MEG. Neurosci Lett 2006;410:31-36.

$\$ 77$ Leirer VM, Wienbruch C, Paul-Jordanov I, Kolassa S, Elbert T, Kolassa IT: Hippocampal activity during the transverse patterning task declines with cognitive competence but not with age. BMC Neurosci 2010;11:1-10.

78 Gramann K, Gwin JT, Bigdely-Shamlo N, Ferris DP, Makeig S: Visual evoked responses during standing and walking. Front Hum Neurosci 2010;4:202.

79 Engel AK, König P, Gray CM, Singer W: Stimulus-dependent neuronal oscillations in cat visual cortex: inter-columnar interaction as determined by cross-correlation analysis. Eur J Neurosci 1990;2:588-606.

80 Gray CM, Viana Di Prisco G: Stimulus-dependent neuronal oscillations and local synchronization in striate cortex of the alert cat. J Neurosci 1997;17:3239-3253.

-81 Lachaux JP, Rodriguez E, Martinerie J, Varela FJ: Measuring phase synchrony in brain signals. Hum Brain Mapp 1999;8:194-208.

-82 Rodriguez E, George N, Lachaux JP, Martinerie J, Renault B, Varela F: Perception's shadow: long-distance synchronization of human brain activity. Nature 1999;397:430-433.

83 Tallon-Baudry C, Bertrand O, Delpuech C, Pernier J: Stimulus specificity of phaselocked and non-phase-locked $40 \mathrm{~Hz}$ visual responses in human. J Neurosci 1996;16: 4240-4249.
84 Treisman A: Feature binding and object perception. Philos Trans R Soc Lond B Biol Sci 1998;353:1295-1306.

85 Sannita WG: Stimulus-related evoked potentials: from basic science to clinical neuropharmacology. Electroencephalogr Clin Neurophysiol Suppl 1996;46:95-106.

86 Blinowska KL: Methods for localization of time-frequency specific activity and estimation of information transfer in brain. Int J Bioelectromagn 2008; 10:2-16.

87 Hassan M, Dufor O, Merlet I, Berrou C, Wendling F: EEG source connectivity analysis: from dense array recordings to brain networks. PLoS One 2014;9:e105041.

88 Stam CJ, van Straaten EC: The organization of physiological brain networks. Clin Neurophysiol 2012;123:1067-1087.

89 Boeijinga PH, Lopes da Silva FH: Differential distribution of beta and theta EEG activity in the entorhinal cortex of the cat. Brain Res 1988;448:272-286.

90 Viriyopase A, Bojak I, Zeitler M, Gielen S: When long-range zero-lag synchronization is feasible in cortical networks. Front Comput Neurosci 2012;6:49.

91 Winterer G, Carver FW, Musso F, Mattay V, Weinberger DR, Coppola R: Complex relationship between BOLD signal and synchronization/desynchronization of human brain MEG oscillations. Hum Brain Mapp 2007;28: 805-816.

92 Alonso JF, Mananas MA, Romero S, Riba J, Barbanoj MJ, Hoyer D: Connectivity analysis of EEG under drug therapy. Proc 29th Annu Int Conf IEEE, Eng Med Biol Soc, Lyon, August 2007, pp 6187-6190.
93 Gianotti LR, Künig G, Lehmann D, Faber PL, Pascual-Marqui RD, Kochi K, Schreiter-Gasser U: Correlation between disease severity and brain electric LORETA tomography in Alzheimer's disease. Clin Neurophysiol 2007; 118:186-196.

94 Verdoorn TA, McCarten JR, Arciniegas DB, Golden R, Moldauer L, Georgopoulos A, Lewis S, Cassano M, Hemmy L, Orr W, Rojas DC: Evaluation and tracking of Alzheimer's disease severity using resting-state magnetoencephalography. J Alzheimer Dis 2011; 26(suppl 3):239-255.

95 Georgopoulos AP, Karageorgiou E, Leuthold AC, Lewis SM, Lynch JK, Alonso AA, Aslam Z, Carpenter AF, Georgopoulos A, Hemmy LS, Koutlas IG, Langheim FJ, McCarten JR, McPherson SE, Pardo JV, Pardo PJ, Parry GJ, Rottunda SJ, Segal BM, Sponheim SR, Stanwyck JJ, Stephane M, Westermeyer JJ: Synchronous neural interactions assessed by magnetoencephalography: a functional biomarker for brain disorders. J Neural Eng 2008;4:349-355.

96 Llinas RR, Ribary U, Jeanmonod D, Kronberg E, Mitra PP: Thalamocortical dysrhythmia: a neurological and neuropsychiatric syndrome characterized by magnetoencephalography. Proc Natl Acad Sci U S A 1999;96:1522215227.

97 Schulman JJ, Cancro R, Lowe S, Lu F, Walton $\mathrm{KD}$, Llinás RR: Imaging of thalamocortical dysrhythmia in neuropsychiatry. Front Hum Neurosci 2011;5:69.

98 Javitt DC, Spencer KM, Thaker GK, Winterer G, Hajós M: Neurophysiological biomarkers for drug development in schizophrenia. Nat Rev Drug Discov 2008;7:68-83. 